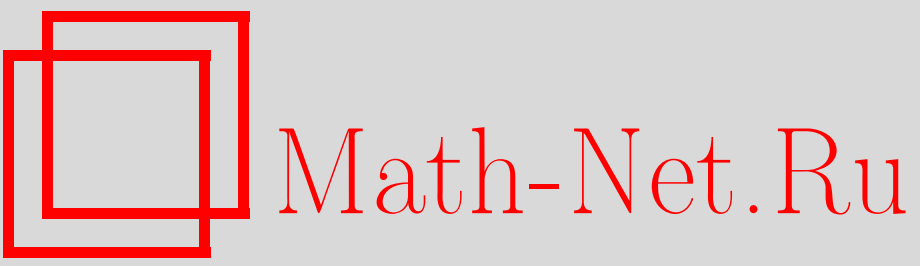

В. И. Кукулин, О. А. Рубцова, Конечномерные аппроксимации операторов теории рассеяния в представлении волновых пакетов, ТМФ, 2004, том 139, номер 2, 291-306

DOI: https://doi.org/10.4213/tmf53

Использование Общероссийского математического портала Math-Net.Ru подразумевает, что вы прочитали и согласны с пользовательским соглашением

http: //www . mathnet.ru/rus/agreement

Параметры загрузки:

IP : 107.22 .136 .117

26 апреля 2023 г., 16:13:30 


\author{
ТЕОРЕТИЧЕСКАЯ \\ И МАТЕМАТИЧЕСКАЯ \\ ФИЗИКА \\ Том 139, № 2 \\ май, 2004
}

(C) 2004 г.

В.И. Кукулин ${ }^{*}$, О. А. Рубцова*

\title{
КОНЕЧНОМЕРНЫЕ АППРОКСИМАЦИИ ОПЕРАТОРОВ ТЕОРИИ РАССЕЯНИЯ В ПРЕДСТАВЛЕНИИ ВОЛНОВЫХ ПАКЕТОВ
}

Рассмотрено несколько типов пакетной дискретизации непрерывного спектра в квантовых задачах теории рассеяния. Подобная дискретизация ведет, как показано авторами ранее, к удобной конечномерной (т.е. матричной) аппроксимации интегральных операторов в теории рассеяния и позволяет свести решение сингулярных интегральных уравнений, отвечающих задаче рассеяния, к удобным чисто алгебраическим уравнениям на аналитическом базисе. При этом все сингулярности выделяются в явном виде. Основное внимание уделяется вопросам практической реализации метода.

Ключевые слова: теория рассеяния, волновой оператор, волновые пакеты, функция Грина, дискретизация континуума.

\section{1. ВВЕДЕНИЕ}

Как хорошо известно, квантовая теория рассеяния [1], [2] может быть наиболее последовательно сформулирована на языке интегральных операторов в гильбертовом (или более общем) пространстве. Основное преимушество такого интегрального подхода заключается в том, что граничные условия уже включены в ядра уравнений, что позволяет сделать естественный переход к случаю рассеяния трех и большего числа частиц. Поэтому язык интегральных операторов в теории рассеяния не только часто используется для формулировки новых подходов, но также очень активно применяется при решении практических задач. Для этих целей интегральные операторы, как правило, заменяют с помощью подходящих квадратурных методов конечномерными аппроксимациями и затем численно решают получившиеся матричные уравнения. Однако на этом пути возникают практические трудности (особенно в многочастичных и многомерных задачах), обусловленные рядом причин.

Во-первых, ядра соответствующих операторов содержат сингулярности, от простых полюсных - в одночастичных задачах, до сложных логарифмических с движушимися

\footnotetext{
${ }^{*}$ Научно-исследовательский институт ядерной физики им. Д. В. Скобельцына, Московский государственный университет, Москва, Россия. E-mail: kukulin@nucl-th.sinp.msu.ru
} 
точками ветвления - в задачах рассеяния трех и большего числа тел. Эти сложные особенности должны как-то учитываться в используемых квадратурных методах. Иначе сходимость соответствующих аппроксимаций к точному решению будет плохой (или вообще будет отсутствовать).

Во-вторых, перевязки между разными наборами координат частиц, неизбежные в многочастичном случае, приводят к появлению в ядрах интегральных операторов громоздких численных матриц преобразования, что заметно усложняет решение соответствуюших матричных уравнений [3], [4]. В итоге практическое решение задачи рассеяния даже в системе трех частиц (например для трех нуклонов) приводит к исключительно сложной вычислительной схеме, требующей применения мощных суперкомпьютеров [5].

Поэтому крайне желательна формулировка альтернативных методов решения многочастичных задач рассеяния, например, с помощью построения особых конечномерных аппроксимаций интегральных операторов, не основанных на обычных численных квадратурах.

Один из таких обших подходов был предложен авторами в работах [6]-[8]. Этот подход основан на формулировке теории рассеяния в терминах чисто дискретных ортонормированных базисов, построенных из стационарных волновых пакетов, принадлежащих пространству $L_{2}$. Тем самым теория рассеяния формулируется в гильбертовом пространстве через конечномерные (т.е. матричные) аналоги интегральных операторов. При этом удалось найти исключительно удобное аналитическое представление для волновых пакетов через суперпозицию хорошо известных в физике собственных функций гармонического осциллятора или суперпозицию гауссовых экспонент. В итоге основные операторы теории рассеяния представляются в виде разложений по простому аналитическому базису с зависящими от энергии коэффициентами [6], [7], которые могут быть найдены из решения простых матричных уравнений как в случае двух частиц, так и в многочастичном случае. Очень важно, что сложная аналитическая зависимость от энергетической переменной оказьвается включенной в эти коэффициенты через довольно простые логарифмические члены. Это сушественно упрошает решение соответствующих уравнений.

В работах [6], [7] мы ввели несколько типов таких пакетных базисов: базис свободных волновых пакетов (отвечающих плоским волнам), базис возмущенных волновых пакетов (отвечаюших точным функциям рассеяния на некотором потенциале), а также несколько типов разложений для ядер операторов. Эти разные типы разложений имеют разные условия применимости, а также разную точность. Поэтому в данной работе, имеюшей практическую направленность, мы решили сравнить между собой различные типы разложений и пакетных базисов.

\section{2. ПРЕДСТАВЛЕНИЕ ВОЛНОВЫХ ПАКЕТОВ}

Поскольку представление волновых пакетов для задачи рассеяния нескольких частиц строится путем прямого умножения (конволюции) соответствуюших пакетов по отдель- 
ным (независимым) координатам Якоби [9], различные типы пакетных представлений удобнее всего исследовать на примере базовой одночастичной задачи [6], [7].

Рассмотрим задачу рассеяния с гамильтонианом

$$
H=H_{0}+V
$$

где $H_{0}$ - оператор кинетической энергии, $V$ - оператор взаимодействия, форма которого не важна для данного рассмотрения. Далее везде мы предполагаем, что значение орбитального момента определено и равно $l$.

В основе метода пакетной дискретизации лежит разбиение непрерывного спектра гамильтонианов $H_{0}$ и $H$ на узкие энергетические полосы (“бины”):

$$
0=\mathcal{E}_{0}<\mathcal{E}_{1}<\cdots<\mathcal{E}_{i}<\cdots<\mathcal{E}_{N}, \quad \Delta_{i}=\mathcal{E}_{i}-\mathcal{E}_{i-1}, \quad \mathcal{E}_{i}^{*}=\frac{1}{2}\left(\mathcal{E}_{i}+\mathcal{E}_{i-1}\right) .
$$

Здесь весь непрерывный спектр ограничивается максимальным значением энергии $\mathcal{E}_{N}$, при этом считается, что $\mathcal{E}_{N} \rightarrow \infty$ при $N \rightarrow \infty$. В каждой такой полосе строятся стационарные волновые пакеты либо из функций свободного движения $\Psi_{0}(E)$, либо из точных функций рассеяния $\Psi(E)$ (предполагается, что и те, и другие нормированы на $\delta$-функцию по энергии),

$$
\begin{aligned}
X_{i}(r) & =\frac{1}{\sqrt{\Delta_{i}}} \int_{\mathcal{E}_{i-1}}^{\mathcal{E}_{i}} \Psi_{0}(E ; r) d E-\text { свободные волновые пакеты (СВП), } \\
Y_{i}(r) & =\frac{1}{\sqrt{\Delta_{i}}} \int_{\mathcal{E}_{i-1}}^{\mathcal{E}_{i}} \Psi(E ; r) d E-\text { возмушенные волновые пакеты (ВВП). }
\end{aligned}
$$

Как легко показать, состояния $\left\{\left|X_{i}\right\rangle\right\}_{i=1}^{N}$ и $\left\{\left|Y_{i}\right\rangle\right\}_{i=1}^{N}$ образуют ортонормированные наборы, поэтому проекционные операторы на подпространства волновых пакетов имеют вид

$$
P_{F}=\sum_{j=1}^{N}\left|X_{j}\right\rangle\left\langle X_{j}\left|, \quad P_{I}=\sum_{j=1}^{N}\right| Y_{j}\right\rangle\left\langle Y_{j}\right| .
$$

Одно из первых упоминаний о стационарных волновых пакетах можно найти в монографии Вигнера [10], где для этих $L_{2}$-векторов используется название "собственные дифференциалы" (или eigendifferentials). Пакетное представление первоначально было использовано для формулировки задачи рассеяния в терминах векторов гильбертова пространства. Наша цель состоит скорее в том, чтобы использовать пакетное представление как удобный и эффективный метод дискретизации континуума, особенно в задачах нескольких тел.

Главное свойство пакетных состояний заключается в том, что их функции являются собственными для соответствуюших спроектированных гамильтонианов, так что имеет место спектральное разложение

$$
P_{F} H_{0} P_{F}=\sum_{i=1}^{N} \mathcal{E}_{i}^{*}\left|X_{i}\right\rangle\left\langle X_{i}\left|, \quad P_{I} H P_{I}=\sum_{i=1}^{N} \mathcal{E}_{i}^{*}\right| Y_{i}\right\rangle\left\langle Y_{i}\right| .
$$


Свойство (6) позволяет провести полную аналогию между “пакетными” состояниями в континууме и состояниями, полученными при диагонализации матрицы гамильтониана в некотором базисе конечной размерности (последние часто используются для численного решения задач). Таким образом, легко найти хорошо сходящиеся разложения СВП и ВВП через какой-либо удобный $L_{2}$-базис (например базис собственных функций гармонического осциллятора или другого подходящего самосопряженного гамильтониана). В итоге удается построить удобные конечномерные аппроксимации основных операторов теории рассеяния в выбранном $L_{2}$-базисе [6]-[8].

Если разложить в определении (4) функцию $|\Psi(E)\rangle$ в ряд Тейлора по энергии в окрестности $E=\mathcal{E}_{i}^{*}$, а затем проинтегрировать по полосе, получим следующее разложение ВВП через производные по энергии от точной волновой функции рассеяния:

$$
\frac{\left|Y_{i}\right\rangle}{\sqrt{\Delta}_{i}}=|\Psi(E)\rangle+\frac{\partial|\Psi(E)\rangle}{\partial E}\left(\mathcal{E}_{i}^{*}-E\right)+\frac{1}{2} \frac{\partial^{2}|\Psi(E)\rangle}{\partial E^{2}}\left\{\left(E-\mathcal{E}_{i}^{*}\right)^{2}+\frac{1}{12}\left(\Delta_{i}\right)^{2}\right\}+\cdots
$$

Когда $E$ принадлежит $i$-му интервалу, т.е. $\left|E-\mathcal{E}_{i}^{*}\right| \leqslant \Delta_{i} / 2$, можно рассматривать (7) как некоторый ряд по параметру $\Delta_{i}$. Заметим, что формально выражение (7) представляет собой бесконечньй ряд по степеням ширины энергетического интервала $\Delta_{i}$. Однако в работе [6] было показано, что в координатном представлении реально возникает ряд по безразмерному параметру $r \delta k_{i}$, где

$$
\delta k_{i}=\sqrt{\frac{m}{2 \mathcal{E}_{i}^{*} \hbar^{2}}} \Delta_{i}
$$

$m$ - приведенная масса частищ в данном канале. Поэтому везде, где мы говорим о малости ширин $\Delta_{i}$, подразумевается, что малы безразмерные величины $r \delta k_{i}$, т.е. получаемые выражения верны в ограниченной области пространства $r \ll R=\delta k_{i}{ }^{-1}$. В этой области можно считать, что собственные дифференциалы $Y_{i}(r)$ отличаются от точных волновых функций рассеяния только нормировочным множителем, причем точность такой аппроксимации имеет порядок малости $\Delta_{i}$,

$$
\frac{Y_{i}(r)}{\sqrt{\Delta_{i}}} \approx \Psi(E ; r)+O\left(\Delta_{i}\right)
$$

Разложение, аналогичное (7), будет верно и для СВП $\left|X_{i}\right\rangle$.

\section{3. ПОСТРОЕНИЕ КОНЕЧНОМЕРНОЙ АППРОКСИМАЦИИ ОПЕРАТОРА ГРИНА}

Для удобства читателя мы помешаем в этом разделе результаты, полученные нами ранее [6]. Однако здесь мы предлагаем более универсальный способ их вьвода.

Мнимая часть оператора Грина в случае простого одноканального рассеяния имеет вид

$$
\operatorname{Im} G_{l}^{(+)}(E)=-i \pi|\Psi(E)\rangle\langle\Psi(E)|
$$


Далее мы будем опускать в обозначении оператора $G$ индексы $l$ и $(+)$, считая, что речь идет о парциальной резольвенте, отвечающей граничному условию на бесконечности в виде расходящихся волн.

Действительную часть оператора Грина можно связать с мнимой посредством дисперсионных соотношений ${ }^{1)}$

$$
\operatorname{Re} G(E)=-\frac{1}{\pi} \mathcal{P} \int d E^{\prime} \frac{\operatorname{Im} G\left(E^{\prime}\right)}{E-E^{\prime}}
$$

Таким образом можно построить конечномерную аппроксимацию оператора $\operatorname{Im} G(E)$ на основе пакетного представления, а затем получить “согласованное" конечномерное представление для действительной части, используя соотношения (10).

Итак, будем далее считать, что $E$ принадлежит некоторому интервалу $\left(\mathcal{E}_{j-1}, \mathcal{E}_{j}\right)$, и называть этот интервал сингулярным. Используя (7), легко вывести разложение для оператора $\left|Y_{j}\right\rangle\left\langle Y_{j}\right|$, которое можно переписать в следуюшем виде:

$$
|\Psi(E)\rangle\langle\Psi(E)|=\frac{\left|Y_{j}\right\rangle\left\langle Y_{j}\right|}{\Delta_{j}}-\frac{\partial[|\Psi(E)\rangle\langle\Psi(E)|]}{\partial E}\left(\mathcal{E}_{j}^{*}-E\right)+O\left(\Delta_{j}^{2}\right)
$$

Согласно (11) можно записать следующую “гистограммную", т.е. конечномерную аппроксимацию для мнимой части оператора Грина (с точностью до величины порядка $\left.O\left(\Delta_{j}\right)\right):$

$$
\operatorname{Im} G^{(1)}(E)=\frac{-i \pi\left|Y_{j}\right\rangle\left\langle Y_{j}\right|}{\Delta_{j}}, \quad E \in\left(\mathcal{E}_{j-1}, \mathcal{E}_{j}\right) .
$$

Подставляя выражение (12) в дисперсионное соотношение (10), получаем следующую конечномерную аппроксимацию для действительной части оператора Грина:

$$
\operatorname{Re} G^{(1)}(E) \approx \sum_{i=1}^{N} \frac{\left|Y_{i}\right\rangle\left\langle Y_{i}\right|}{\Delta_{i}} \ln \left|\frac{\mathcal{E}_{i-1}-E}{\mathcal{E}_{i}-E}\right|
$$

Легко видеть, что (12) и (13) отвечают проекции полного оператора Грина на подпространство волновых пакетов, т.е. $G^{(1)}(E)=P_{I} G_{l}^{(+)}(E) P_{I}$. Такая аппроксимация в работе [7] была дополнительно упрошена заменой непрерьвного энергетического спектра гамильтониана дискретным набором точек $\left\{\mathcal{E}_{i}^{*}\right\}_{i=1}^{N}$ (средних точек бинов). Расчеты наблюдаемых величин (фаз рассеяния) в этих дискретных точках, основанные на представлении (13), привели к достаточно точным значениям. Для дальнейшего уточнения этих результатов нужно учесть более высокие порядки разложения (11).

\footnotetext{
1) Для простоты будем считать, что связанные состояния в системе отсутствуют.
} 
В работе [6] было показано, что конечномерный оператор $\widehat{D}_{i}$, определяемый следующим образом:

$$
\widehat{D}_{i}= \begin{cases}\frac{\left|Y_{i}\right\rangle\left\langle Y_{i}\right|}{\Delta_{i}}-\frac{\left|Y_{i-1}\right\rangle\left\langle Y_{i-1}\right|}{\Delta_{i-1}}, & i=N, \\ \frac{1}{2}\left\{\frac{\left|Y_{i+1}\right\rangle\left\langle Y_{i+1}\right|}{\Delta_{i+1}}-\frac{\left|Y_{i-1}\right\rangle\left\langle Y_{i-1}\right|}{\Delta_{i-1}}\right\}, & i=2, \ldots, N-1, \\ \frac{\left|Y_{i+1}\right\rangle\left\langle Y_{i+1}\right|}{\Delta_{i+1}}-\frac{\left|Y_{i}\right\rangle\left\langle Y_{i}\right|}{\Delta_{i}}, & i=1,\end{cases}
$$

является аппроксимацией билинейной формы

$$
\left[\frac{\partial|\Psi(E)\rangle\langle\Psi(E)|}{\partial E}\right]_{E=\mathcal{E}_{i}^{*}} \Delta_{i}
$$

с точностью до малых второго порядка относительно ширины бинов. Таким образом, можно записать соотношение, аналогичное (11),

$$
\begin{aligned}
|\Psi(E)\rangle\langle\Psi(E)|= & \frac{\left|Y_{j}\right\rangle\left\langle Y_{j}\right|}{\Delta_{j}}+\widehat{D}_{j}\left(E-\mathcal{E}_{j}^{*}\right)+\frac{1}{2} \frac{\partial^{2}[|\Psi(E)\rangle\langle\Psi(E)|]}{\partial E^{2}}\left(E-\mathcal{E}_{j}^{*}\right)^{2}- \\
& -\frac{1}{24}\left\{\frac{\partial^{2}|\Psi(E)\rangle}{\partial E^{2}}\langle\Psi(E)|+| \Psi(E)\rangle \frac{\partial^{2}\langle\Psi(E)|}{\partial E^{2}}\right\} \Delta_{j}^{2}+O\left(\left(\Delta_{j}\right)^{3}\right) .
\end{aligned}
$$

Заметим, что последнее выражение верно при том условии, что разбиение спектра локально мало отличается от эквидистантного, т.е. $\left|\Delta_{j+1}-\Delta_{j}\right| \ll \Delta_{j}$.

Итак, поправка к выражению (12), имеюшая порядок малости $O\left(\Delta_{j}^{2}\right)$, имеет вид

$$
\operatorname{Im} G^{(2)}(E)=-i \pi \widehat{D}_{j} \frac{E-\mathcal{E}_{j}^{*}}{\Delta_{j}} .
$$

Для действительной части оператора Грина на основе дисперсионного представления получаем следуюшую поправку второго порядка:

$$
G^{(2)}(E) \approx-\sum_{i=1}^{N} \widehat{D}_{i}\left[1-\frac{\left(E-\mathcal{E}_{i}^{*}\right)}{\Delta_{i}} \ln \left|\frac{\mathcal{E}_{i-1}-E}{\mathcal{E}_{i}-E}\right|\right] .
$$

По-видимому, можно построить и поправки более высокого порядка, используя конечномерные аппроксимации для высших производных по энергии от точных функций рассеяния.

Запишем окончательно полученную конечномерную аппроксимацию оператора Грина в представлении волновых пакетов в следуюшем виде:

$$
\widetilde{G}(E)=G^{(1)}(E)+G^{(2)}(E),
$$

где $G^{(1)}(E) \sim O\left(\Delta_{i}\right), G^{(2)}(E) \sim O\left(\Delta_{i}^{2}\right)$ как для действительной, так и для мнимой частей оператора Грина. Член $G^{(2)}(E)$ будем называть поправочным. 
Более точная оценка величины поправочного члена. Как уже упоминалось выше, "гистограммная" аппроксимация $G^{(1)}(E)$ возникает при нахождении наблюдаемых в точках “дискретизованного" спектра $\mathcal{E}_{i}^{*}$, т.е. в средних точках бинов $\Delta_{i}$. Оценим величину поправки $G^{(2)}$ при этих значениях энергии. Пусть $E=\mathcal{E}_{j}^{*}$. Запишем следуюшие разложения логарифмических функций:

$$
\begin{aligned}
& \ln \left|\frac{\mathcal{E}_{i-1}-E}{\mathcal{E}_{i}-E}\right|=\ln \left|\frac{\mathcal{E}_{i}^{*}-\frac{1}{2} \Delta_{i}-E}{\mathcal{E}_{i}^{*}+\frac{1}{2} \Delta_{i}-E}\right|= \\
& = \begin{cases}\frac{\Delta_{i}}{E-\mathcal{E}_{i}^{*}}\left\{1+\frac{1}{12}\left(\frac{\frac{1}{2} \Delta_{i}}{E-\mathcal{E}_{i}^{*}}\right)^{2}+O\left(\left[\frac{\Delta_{i}}{E-\mathcal{E}_{i}^{*}}\right]^{4}\right)\right\}, & \left|E-\mathcal{E}_{i}^{*}\right|>\frac{1}{2} \Delta_{i}, \\
4 \frac{E-\mathcal{E}_{i}^{*}}{\Delta_{i}}\left\{1+\frac{1}{12}\left(\frac{E-\mathcal{E}_{i}^{*}}{\frac{1}{2} \Delta_{i}}\right)^{2}+O\left(\left[\frac{E-\mathcal{E}_{i}^{*}}{\Delta_{i}}\right]^{4}\right)\right\}, & \left|E-\mathcal{E}_{i}^{*}\right|<\frac{1}{2} \Delta_{i} .\end{cases}
\end{aligned}
$$

Из этих разложений легко получается и разложение для функции

$$
1-\frac{\left(E-\mathcal{E}_{i}^{*}\right)}{\Delta_{i}} \ln \left|\frac{\mathcal{E}_{i-1}-E}{\mathcal{E}_{i}-E}\right| .
$$

В результате, учитывая в упомянутых выше разложениях только квадратичные порядки малости, можно записать

$$
\begin{aligned}
\operatorname{Re} \widetilde{G}(E) \approx & \sum_{\substack{i=1 \\
i \neq j}}^{N}\left\{\frac{\left|Y_{i}\right\rangle\left\langle Y_{i}\right|}{\Delta_{i}} \frac{\Delta_{i}}{E-\mathcal{E}_{i}^{*}}-\widehat{D}_{i}\left(\frac{\Delta_{i}}{E-\mathcal{E}_{i}^{*}}\right)^{2}\right\}+ \\
& +4 \frac{\left|Y_{j}\right\rangle\left\langle Y_{j}\right|}{\Delta_{j}} \frac{E-\mathcal{E}_{i}^{*}}{\Delta_{i}}-\widehat{D}_{j}\left\{1-4\left(\frac{E-\mathcal{E}_{i}^{*}}{\Delta_{i}}\right)^{2}\right\} .
\end{aligned}
$$

Из этого приближенного равенства видно, что основной вклад в поправку второго порядка $G^{(2)}(E)$ дает слагаемое на сингулярном интервале (т.е. при $\left|E-\mathcal{E}_{j}^{*}\right|<\Delta_{j} / 2$ ). Учитывая лишш основные слагаемые при $E=\mathcal{E}_{j}^{*}$, получаем простое выражение для конечномерной аппроксимации действительной части оператора Грина,

$$
\operatorname{Re} \widetilde{G}(E) \approx \sum_{\substack{i=1 \\ i \neq j}}^{N} \frac{\left|Y_{i}\right\rangle\left\langle Y_{i}\right|}{E-\mathcal{E}_{i}^{*}}-\widehat{D}_{j},
$$

где оператор $\widehat{D}_{j}$ определяется согласно (14).

Итак, с учетом "основных порядков малости" видно, что поправка второго порядка $G^{(2)}$ существенна главным образом на сингулярном интервале $\left(\mathcal{E}_{j-1}<E<\mathcal{E}_{j}\right)$ и что ее величина в средней точке равна $G^{(2)}\left(\mathcal{E}_{j}^{*}\right)=-\widehat{D}_{j}$. Если далее использовать для этого оператора определение (14), представление (22) будет практически точно совпадать с аппроксимацией оператора Грина, полученной в работе [8] на основе качественных соображений.

Таким образом, мы нашли для операторной функции Грина в обшем виде конечномерное представление, справедливое с точностью $O\left(\Delta_{i}^{2}\right)$. На основе этого представления обшего вида можно элементарным образом построить весьма точные аналитические конечномерные аппроксимации для резольвенты путем простой диагонализации свободного или полного гамильтониана на подходящем $L_{2}$-базисе. 


\section{4. КОНЕЧНОМЕРНЫЕ АППРОКСИМАЦИИ Т- И Л-ОПЕРАТОРОВ}

На основе полученных выше конечномерных представлений для оператора Грина легко построить дискретные аналоги других операторов теории рассеяния. В частности, для нахождения наблюдаемых величин удобно использовать $T$-оператор. В рамках метода пакетной дискретизации континуума конечномерный аналог этого оператора можно построить двумя способами на основе либо возмущенных, либо свободных волновых пакетов.

4.1. Представление возмущенных волновых пакетов. В качестве основного базиса системы будем использовать набор ВВП $\left\{\left|Y_{i}\right\rangle\right\}_{i=1}^{N}$. Тогда для нахождения $T$-оператора достаточно подставить одну из конечномерных аппроксимаций оператора Грина в известное тождество

$$
T(E)=V+V G^{(+)}(E) V
$$

Далее элементы $T$-матрицы на массовой поверхности и вне ее находятся по определению

$$
T_{l}\left(E_{1}, E_{2} ; E\right)=\left\langle\Psi_{0}\left(E_{1}\right)|T(E)| \Psi_{0}\left(E_{2}\right)\right\rangle .
$$

В качестве ВВП-состояний можно использовать собственные функции матрицы гамильтониана в некотором $L_{2}$-базисе, если параметры разбиений будут совпадать (см. детали в работе [6]).

Описанный здесь метод нахождения наблюдаемых величин с использованием ВВПпредставления кажется очень удобным. В самом деле, он позволяет решать множество типов задач рассеяния для эрмитовых гамильтонианов, например для сложных нелокальных операторов взаимодействия, путем простой диагонализации матрицы гамильтониана в подходящем $L_{2}$-базисе (см. [6] и ниже). Однако в случае неэрмитовых операторов взаимодействия этот метод вряд ли применим. В последнем случае удобнее использовать аппроксимации свободной резольвенты в базисе свободных волновых пакетов.

4.2. Представление свободных волновых пакетов. В работе [7] было показано, что дискретные аналоги основных операторов теории рассеяния и дискретизованные функции рассеяния (т.е. стационарные волновые пакеты) можно построить также в базисе СВП $\left\{\left|X_{i}\right\rangle\right\}_{i=1}^{N}$. В таком подходе в качестве "краеугольного камня" используется конечномерная аппроксимация свободной резольвенты. Последняя подставляется в уравнение Липпмана-Швингера для $T$-оператора

$$
T(E)=V+V G_{0}^{(+)}(E) T(E)
$$

В результате получается простое алгебраическое уравнение для матрицы дискретизованного оператора $P_{I} T P_{I}$, решением которого является матрица

$$
T=\Omega V
$$


где $\Omega=\left(1-V G_{0}(E)\right)^{-1}$ представляет собой конечномерный аналог волнового оператора Меллера (подробно см. в [6]).

В работе [6] в качестве конечномерной аппроксимации для операторной функции Грина использовался спроектированный оператор свободной резольвенты $G_{0}^{(1)}=P_{F} G_{0} P_{F}$ (при этом наблюдаемые величины в точности отвечают рассеянию на потенциале $\left.P_{F} V P_{F}\right)$. В дальнейшем можно улучшить аппроксимацию операторов рассеяния, если использовать представление для свободной резольвенты в виде

$$
\begin{aligned}
\widetilde{G}_{0}(E)= & \sum_{i=1}^{N}\left\{\frac{\left|X_{i}\right\rangle\left\langle X_{i}\right|}{\Delta_{i}} \ln \left|\frac{\mathcal{E}_{i-1}-E}{\mathcal{E}_{i}-E}\right|-\widehat{D}_{0 i}\left[1-\frac{\left(E-\mathcal{E}_{i}^{*}\right)}{\Delta_{i}} \ln \left|\frac{\mathcal{E}_{i-1}-E}{\mathcal{E}_{i}-E}\right|\right]\right\}- \\
& -i \pi\left\{\frac{\left|X_{j}\right\rangle\left\langle X_{j}\right|}{\Delta_{j}}+\widehat{D}_{0 j} \frac{\left(E-\mathcal{E}_{j}^{*}\right)}{\Delta_{j}}\right\} \text { при } E \in\left(\mathcal{E}_{j-1}, \mathcal{E}_{j}\right) .
\end{aligned}
$$

Здесь операторы для поправок $\widehat{D}_{0 i}$ определяются формулой $(14)$, в которой состояния $\left|Y_{i}\right\rangle$ заменены на $\left|X_{i}\right\rangle$.

Удобством представления в базисе свободных волновых пакетов является его полная универсальность и применимость как для аппроксимации эрмитовых, так и для неэрмитовых или даже зависящих явно от энергии операторов взаимодействия. В последнем случае $V=V(E)$ и $\Omega=\left(1-V(E) G_{0}(E)\right)^{-1}$

Однако платой за универсальность является большая трудоемкость численного решения задачи, которая в данном случае требует многократного обрашения матришы $\left(1-V G_{0}(E)\right)$ при всех требуемых энергиях. Здесь уместно напомнить, что построение $T$-матрицы через ВВП при всех энергиях требует всего лиш однократной диагонализации матрицы гамильтониана на выбранном $L_{2}$-базисе.

Возможно использовать гибридный подход в тех случаях, когда основная часть оператора Гамильтона является эрмитовым оператором, а неэрмитова (или зависящая от энергии) часть может рассматриваться как возмущение $H^{\prime}$. В этом случае для эрмитовой части строится представление ВВП (через диагонализацию эрмитовой части гамильтониана), а для неэрмитовой добавки $H^{\prime}$ можно построить ряд теории возмушений в пакетном представлении (т.е. также в простой матричной форме [6]). Такой гибридньй подход должен быть очень удобным в многоканальных задачах рассеяния, в которых связь остальных каналов с упругим (или каким-то выделенным) каналом является достаточно слабой. При этом исключение неупругих каналов дает, как хорошо известно [11], явно зависящую от энергии нелокальную добавку $\delta V(E)$ к прямому взаимодействию в упругом канале $V_{\text {dir }}$. Именно такую структуру имеют все операторы так называемого поляризационного взаимодействия составных частиц, возникающего из-за учета виртуальных переходов взаимодействующих частиц в свои возбужденные состояния или в промежуточные возбужденные состояния системы как целого, в атомной, молекулярной и ядерной физике. 


\section{5. ПРИМЕРЫ}

В этом разделе мы на ряде простых примеров продемонстрируем скорость сходимости обсужденных выше конечномерных аппроксимаций операторов резольвенты и $T$-матрицы, а также удобство развитой схемы.

5.1. Конкретные реализации СВП- и ВВП-базисов. Как было сказано вьше, для решения задачи рассеяния на центральном потенциале можно использовать либо СВП-, либо ВВП-базисы, при этом в случае неэрмитовых потенциалов предпочтительнее использовать базис СВП. Для иллюстрации и сравнения точности разных представлений мы вычислили $S$-волновые фазовые сдвиги рассеяния на потенциале гауссова типа на основе этих двух подходов. Волновые пакеты строились путем диагонализации матриц свободного и полного гамильтонианов в одном и том же $L_{2}$-базисе. В качестве такого базиса был выбран ортогональный базис волновых функций гармонического осциллятора. Свойства базиса зависят от радиального параметра $r_{\omega}=\sqrt{\hbar / \mu \omega}$, который подбирается так, чтобы сходимость к точному решению была наилучшей (в случае низкоэнергетического рассеяния значение $r_{\omega}$ легко находится из принципа Ритца, т.е. из условия минимума энергии связанных состояний). На рис. 1 приведены зависимости от энергии фазовых сдвигов, полученных в ВВП-базисе (т.е. при использовании лишь однократной диагонализации матрицы полного гамильтониана) для разного числа базисных функций $N$. Расчеты проводились в средних точках энергетических интервалов. Обозначения G1 и G2 на рисунке отвечают расчетам на основе гистограммной аппроксимации оператора Грина и по формуле с учетом поправок второго порядка, соответственно, $V_{0}$ - глубина потенциальной ямы.

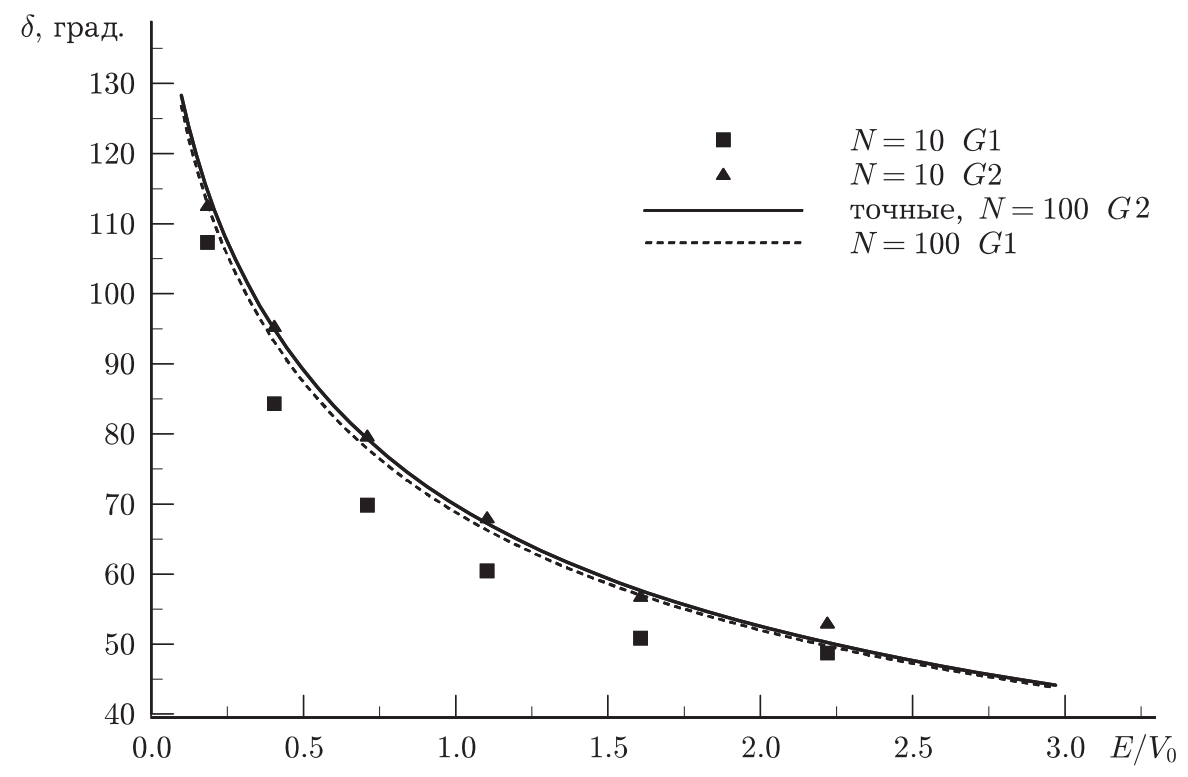

Рис. 1 
Расчеты тех же фазовых сдвигов в СВП-базисе в средних точках энергетических интервалов привели к практически таким же результатам (поэтому мы не приводим для них отдельного рисунка). Сравнительный анализ точности нахождения фаз рассеяния в СВП- и ВВП-подходах приведен на рис. 2. При этом в качестве “точных" значений использовались фазовые сдвиги, полученные путем прямого численного интегрирования уравнения Шредингера. На рис. 2а приведены относительные погрешности фаз рассеяния $\alpha$, полученных с использованием гистограммной аппроксимации как в ВВП-, так и СВП-подходах для разного числа базисных функций. На рис. 26 приведены относительные погрешности фаз, найденных с использованием поправочного члена второго порядка. Как хорошо видно из приведенных рисунков, точность расчетов в СВП- и ВВП-подходах практически совпадает (метод СВП дает несколько лучшие результаты). Важно отметить, что учет поправочных членов позволяет улучшить точность вычислений фазовых сдвигов на порядок величины.

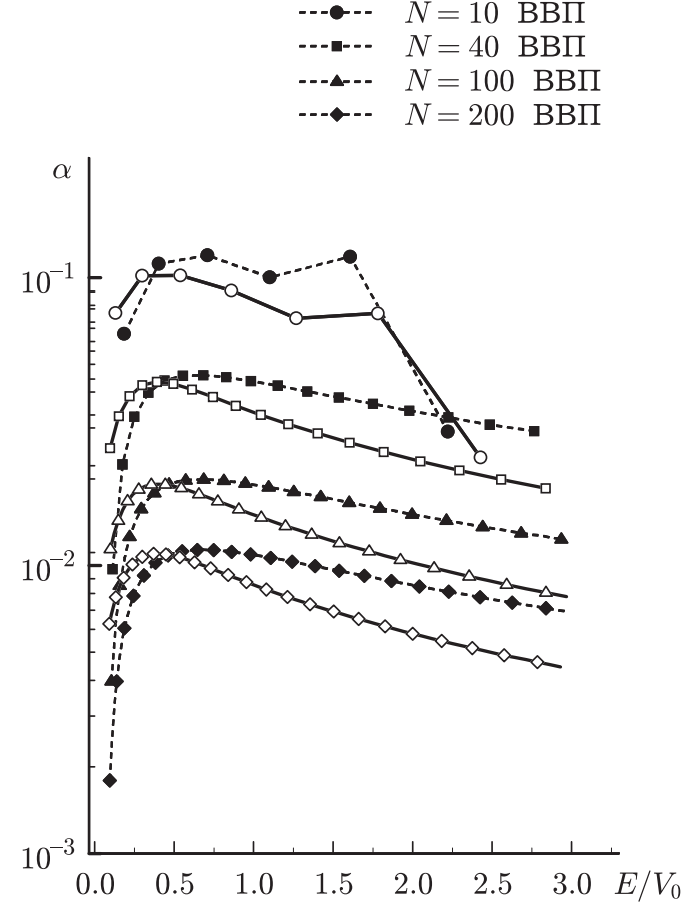

a
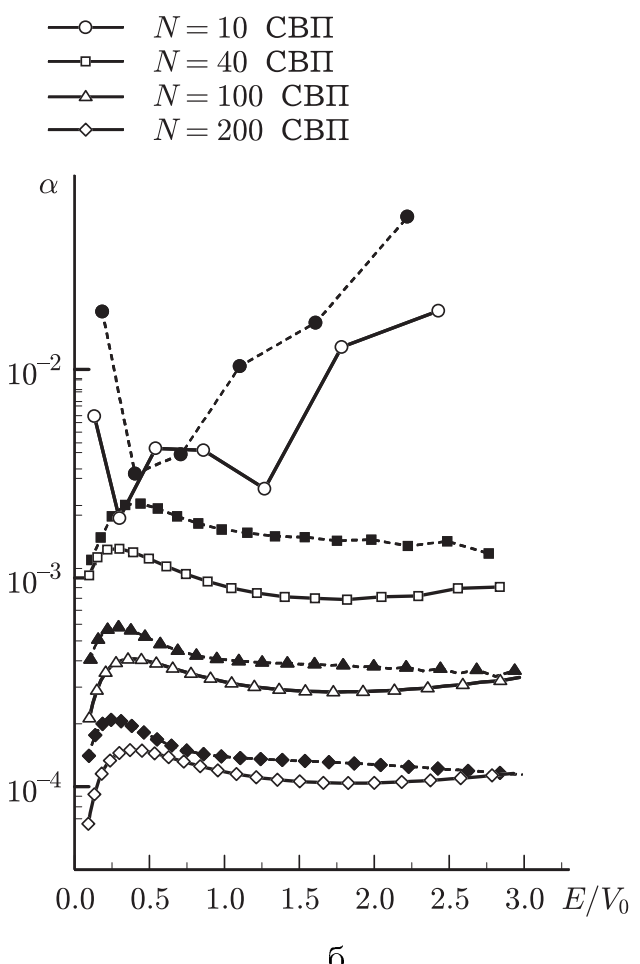

б

Рис. 2

Следует подчеркнуть, что построенная в СВП-представлении $T$-матрица удовлетворяет точному условию унитарности (при расчетах в средних точках бинов), в то время как $T$-матрица, найденная в ВВП-представлении, этому условию не удовлетворяет.

Подводя итог, следует отметить, что расчеты даже с простой гистограммной аппрок- 
симацией приводят к ошибкам порядка нескольких процентов при весьма скромной размерности базиса $N=20$. А при размерности базиса $N \simeq 50-60$ ошибка в фазовом сдвиге составляет уже $0.1 \%$. Это весьма важно для обобшения метода пакетной дискретизации на задачу рассеяния нескольких тел, где проективный метод позволяет значительно упростить решение [9]. Более того, исключение вклада закрытых каналов, как показано ниже, позволяет дополнительно уменьшить размерность базиса в 2-3 раза!

Энергетическое поведение конечномерной аппроксимации для резольвенты. Легко видеть, что построенный конечномерный оператор Грина $\widetilde{G}(E)$ не является непрерывной по энергии функцией. В граничных точках интервалов ее действительная часть имеет логарифмические разрывы, а мнимая - скачки. Однако при вычислениях для некоторой фиксированной энергии эти трудности легко обойти путем увеличения числа базисных функций (при этом положения "особых" точек сдвигаются). Для иллюстрации мы разделили весь энергетический спектр в интервале $(0.1-3.0) V_{0}$ на 100 субинтервалов равной ширины и вычислили фазовые сдвиги в каждой "граничной" точке. Результаты вычислений в ВВП-базисе по формуле с поправкой второго порядка представлены на рис. 3. Как хорошо видно, при выбранном шаге вычислений достаточно использовать базис всего из 40 функций для получения весьма “гладкой” кривой фазовых сдвигов, практически совпадающей с кривой точных значений.

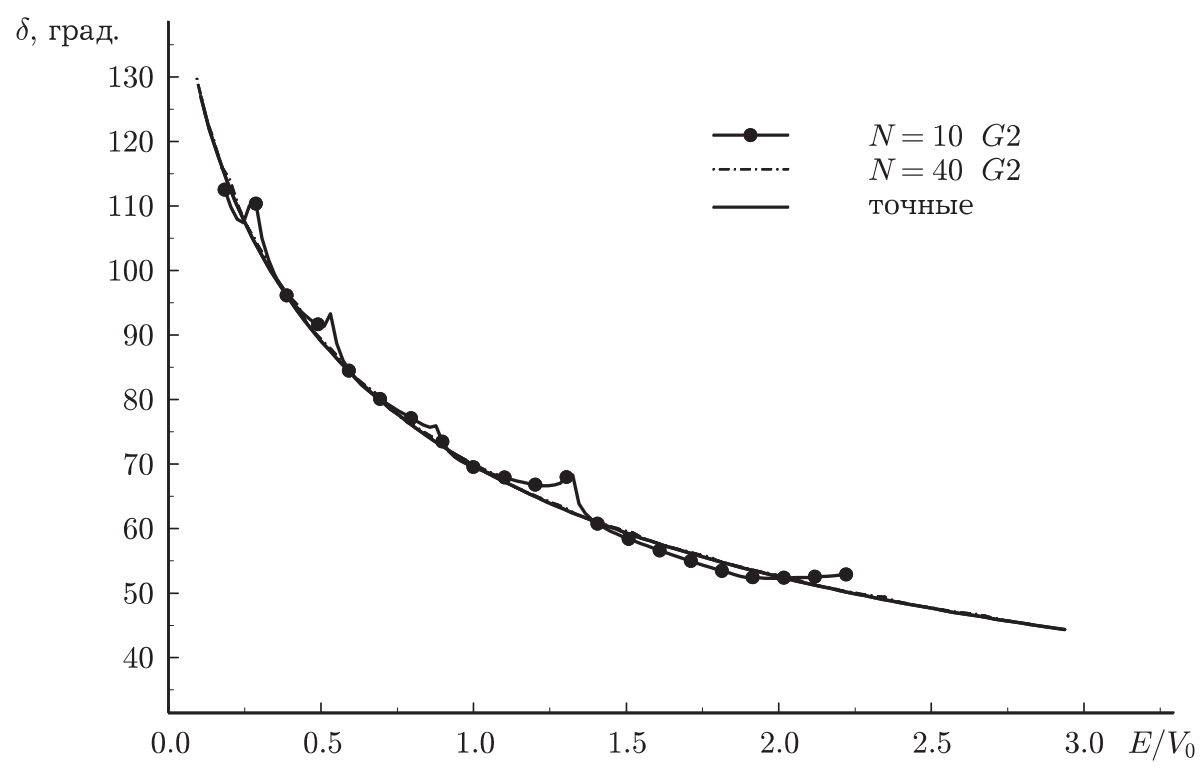

Рис. 3

5.2. Исследование сходимости при фиксированной энергии. Влияние закрытых каналов. Построенные конечномерные аппроксимации для действительных частей полной $\widetilde{G}(E)$ или свободной $\widetilde{G}_{0}(E)$ резольвент представляют собой спектральные разложения по "полному спектру" ВВП- или СВП-состояний, соответственно. 
При этом сумма по тем энергетическим интервалам, которые лежат выше сингулярного (т.е. того интервала, которому принадлежит выбранное значение энергии, будем считать его $j$-м), представляет собой сумму по закрытым каналам. Вполне естественно допустить, что вклад от каждого бина, представляющего закрытый канал, будет заметно уменьшаться с удалением от сингулярного интервала. Таким образом, при выбранном значении энергии можно будет учесть лишь вклад нескольких таких закрытых каналов, непосредственно примыкающих к порогу, т.е. заменить полную спектральную сумму для резольвенты, состоящую из $N$ слагаемых, суммой из $M$ слагаемых, где $j<M \leqslant N$.

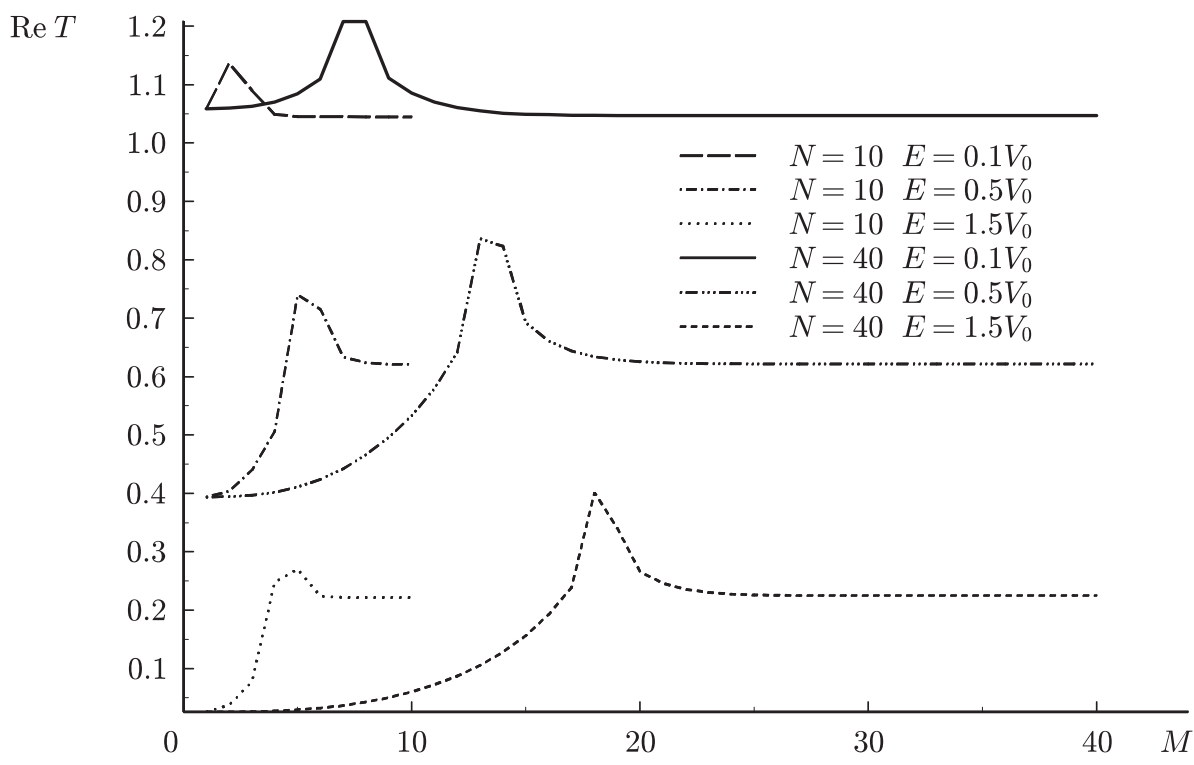

Рис. 4

Для проверки этих предположений мы провели детальные расчеты элементов $T$-матрицы на массовой поверхности и связанных с ними фазовых сдвигов при трех значениях энергии: $E_{1}=0.1 V_{0}, E_{2}=0.5 V_{0}$ и $E_{3}=1.5 V_{0}$ (где $V_{0}$ - параметр глубины тестового потенциала). Наиболее наглядно картина сходимости относительно вклада закрытых каналов видна при расчетах в ВВП-представлении, поскольку $T$-матрица в этом случае представляется в виде простой спектральной суммы. На рис. 4 представлены зависимости действительной части элементов $T$-матрицы на массовой поверхности, найденных в ВВП-представлении, от числа $M$ учитываемых бинов для трех разных значений энергии (малого, промежуточного и достаточно высокого) и двух размерностей базисов $\mathrm{N}=10$ и 40. Как хорошо видно, графики выходят на "плато" достаточно быстро с увеличением $M$.

Мы также провели исследование величины вклада закрытых каналов в СВП-представлении. На рис. 5 приведены кривые “сходимости” фазовых сдвигов по числу учитываемых каналов $M$ для значений энергии $E=0.1 V_{0}$ и $E=0.5 V_{0}$ в ВВП- и СВП- 


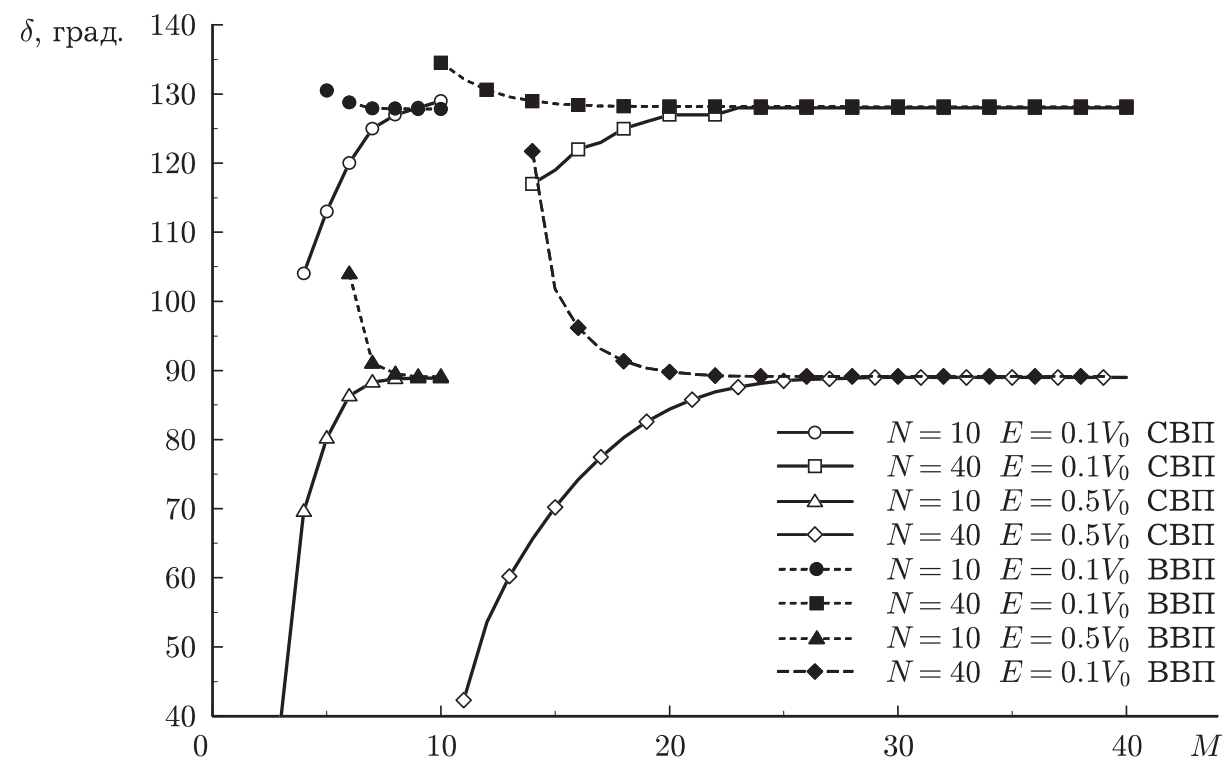

Рис. 5

представлениях. Легко видеть, что сходимость для ВВП-представления оказывается более быстрой. Кроме того, в последнем случае наблюдается сходимость фазовых сдвигов “сверху", в то время как в СВП-представлении сходимость будет “снизу".

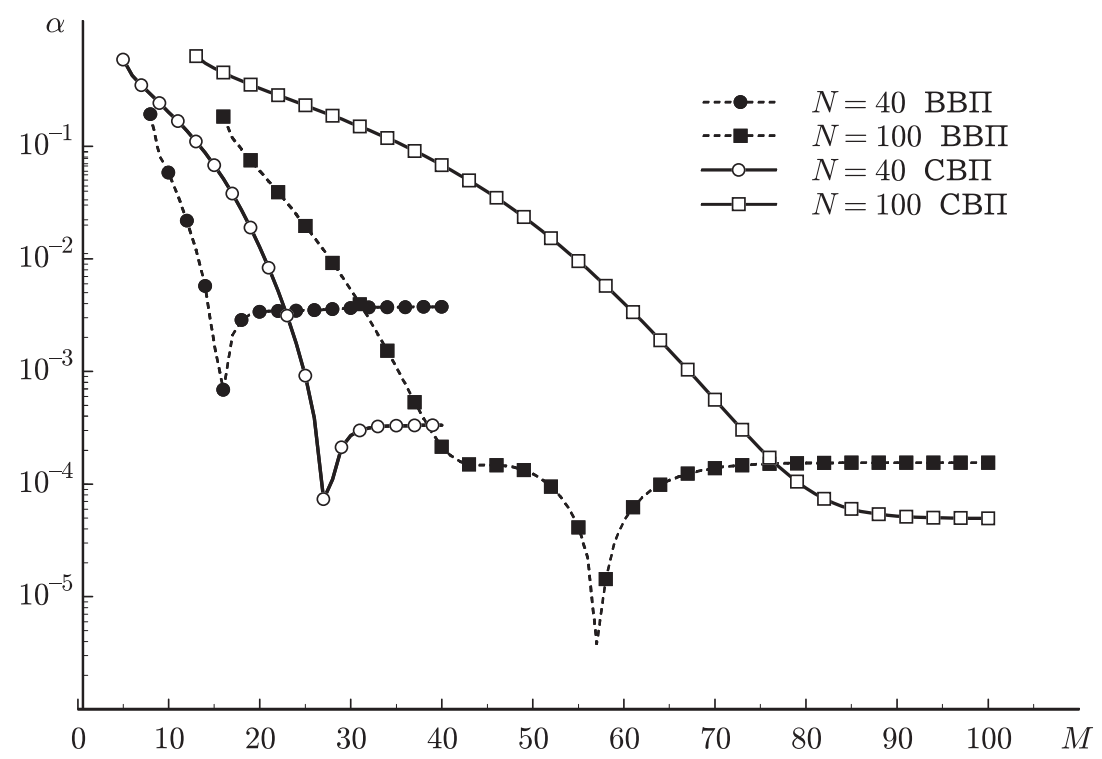

Рис. 6 
На рис. 6 приведены зависимости абсолютных величин относительных погрешностей $\alpha$ фазовых сдвигов от числа учитываемых каналов $M$ при малой энергии $E=0.1 V_{0}$ для СВП- и ВВП-базисов при полной размерности $N=40$ и 100.

Анализируя приведенные графики, можно сделать вывод, что в ВВП-представлении достаточно учитывать вклад лишь от половины или даже одной трети (в зависимости от значений $N$ и $E$ ) полного числа энергетических полос, что позволяет заметно ускорить процесс вычислений. В СВП-представлении “отбрасывание лишних вкладов" связано с потерей точности, однако, если ограничиться точностью порядка нескольких десятых процента, то и в этом случае можно заметно сократить размерность матриц при обращении. При этом выигрыш будет многократно расти в задачах многочастичного рассеяния, где размерности базисов по каждой независимой координате Якоби будут перемножаться. Таким образом, уменьшение базиса в три раза в одночастичном (и двухчастичном) рассеянии приводит к сокрашению размера пакетного базиса почти на порядок(!) в задачах трехчастичного рассеяния.

\section{6. ЗАКЛЮЧЕНИЕ}

В данной работе мы детально исследовали различные типы пакетной дискретизации как универсальные методы построения конечномерной аппроксимации основных операторов теории рассеяния и сравнили их между собой. Это сравнение показало, что пакетные базисы СВП и ВВП дают примерно одинаковую точность представления для операторной резольвенты. При этом базис ВВП предпочтительнее использовать для любых эрмитовых операторов взаимодействия, тогда как базис СВП хорошо работает как для эрмитовых, так и для неэрмитовых гамильтонианов.

Мы также развили обший метод нахождения поправок высших порядков (относительно ширин энергетических полос $\Delta$ ) к оператору Грина на основе дисперсионных соотношений. Было, в частности, показано, что учет поправки второго порядка позволяет на порядок увеличить точность вычисления наблюдаемых. Однако дальнейшее увеличение точности вычислений за счет поправок более высоких порядков хотя и возможно, но, по-видимому, нецелесообразно. Удобнее достигать более высокой точности либо путем увеличения размерности базиса $N$ (см. рис. 1 и 3 ), либо выбором более подходящего $L_{2}$-базиса для представления волновых пакетов. В этой связи весьма обещающим кажется недавно разработанный авторами базис гибридного типа [12], который представляет собой среднее между обычным одночастотным осцилляторным базисом и мультимасштабным гауссовым базисом (многочастотный осцилляторный базис).

Здесь уместно подчеркнуть, что при всем удобстве дискретного пакетного представления для задач потенциального рассеяния различного типа основные преимушества такого подхода по сравнению с традиционными методами аппроксимации интегральных операторов в теории рассеяния будут проявляться в многомерных и многочастичных задачах, где пакетная техника допускает прямое обобщение, сохраняя свою простоту и аналитический характер [9].

5 Теоретическая и математическая физика, т. 139, № 2, 2004 г. 
Благодарности. Авторы выражают глубокую благодарность редактору английского издания ТМФ Вильяму Эверетту за ряд полезных замечаний, относящихся к терминологии, а также за важные ссылки на работы, связанные с нашим подходом. Работа выполнена при частичной финансовой поддержке РФФИ (гранты № 01-02-16621 и 03-02-06506).

\section{Список литературы}

[1] Р. Ньютон. Теория рассеяния волн и частиц. М.: Мир, 1969.

[2] М. Гольдбергер, К. Ватсон. Теория столкновений. М.: Мир, 1967.

[3] Э. Шмид, Х. Цигельман. Проблема трех тел в квантовой механике. М.: Наука, 1979.

[4] Y. E. Kim, A. Tubis. Phys. Rev. C. 1973. V. 7. P. 1710.

[5] W. Glöckle, H. Witala, D. Hüber, H. Kamada, J. Golak. Phys. Rep. 1996. V. 274. P. 107.

[6] В. И. Кукулин, О. А. Рубцова. ТМФ. 2002. Т. 130. С. 64.

[7] В. И. Кукулин, О. А. Рубцова. ТМФ. 2003. Т. 134. С. 460.

[8] О. А. Рубцова, В. И. Кукулин. ЯФ. 2001. Т. 64. С. 1882.

[9] O. A. Rubtsova, V. I. Kukulin. Efficient technique for solving few-body scattering problems by the wave-packet continuum discretization. In: Few-Body Problems in Physics'02 (Few-Body Systems Supplement. V. 14). Eds. R. Krivec, B. Golli, M. Rosina, S. Širca. Wien: Springer, 2003. P. 211.

[10] E. Вигнер. Теория групп и ее приложение к квантовомеханической теории атомных спектров. М.: ИЛ, 1961.

[11] Дж. Тейлор. Теория рассеяния. Квантовая теория нерелятивистских столкновений. М.: Мир, 1975.

[12] О. А. Рубцова, В. И. Кукулин, С. А. Никольчинкина. Построение оптимальных вариационных базисов для расчета спектров квантовых систем. ЯФ. (в печати).

Поступила в редакцию $10 . \mathrm{IV} .2003$ г. 